\title{
Resilience Factors Important in Health-Related Quality of Life of Subjects With COPD
}

\author{
Danielle L Cannon PhD, Krishna Bajee Sriram MBBS FRACP PhD, Alan Wee-Chung Liew PhD, \\ and Jing Sun PhD
}

\begin{abstract}
BACKGROUND: Common among patients with COPD is declining health-related quality of life (HRQOL). Although results of research identified some factors associated with HRQOL, resilience factors are yet to be fully investigated. METHODS: This study examined resilience and demographic factors associated with HRQOL. Participants $>40 \mathrm{y}$ old were recruited from community health programs and hospitals in South East Queensland. Self-administered questionnaires were used to query subjects' HRQOL and levels of resilience. A decision tree examined the factors important to HRQOL in 159 subjects with COPD. RESULTS: Factors of importance in the HRQOL of subjects with COPD were found in 3 domains of the St George Respiratory Questionnaire. Of importance on the breathlessness domain was marital status, defensive coping, coping, number of comorbidities, relationships, decision-making, self-esteem, self-efficacy, and professional support of health and well-being. Of the symptoms domain, self-efficacy, recruitment location, anxiety/depression, decision-making, self-esteem, coping, relationships, professional support of health and wellbeing, and risks were important. The cough domain found recruitment location, anxiety/depression, professional support of health and well-being, coping, and defensive coping to be important for subjects' HRQOL. CONCLUSIONS: Resilience and confounding factors were of importance in the HRQOL of subjects with COPD. Thus, consultation with a medical professional, especially at discharge, who identifies, encourages, and approves of the patient's disease management abilities will enhance both resilience and HRQOL. Key words: COPD; resilience; self-esteem; defensive coping; professional support; hospitalization; HRQOL. [Respir Care 2018;63(10):1281-1292. (C) 2018 Daedalus Enterprises]
\end{abstract}

\section{Introduction}

COPD is a preventable and treatable illness of the lungs, characterized by persistent air-flow limitation, hyperinflation, abnormal gas exchange, mucus hypersecretion, pulmonary hypertension, exacerbations, and other systemic problems, (eg, cardiac issues and frailty syndrome). ${ }^{1-3}$ Due to the dis-

Dr Cannon is affiliated with Menzies Health Institute Queensland and School of Medicine, Griffith University, Southport, Gold Coast, Australia. Dr Sriram is affiliated with the Department of Respiratory Medicine, Gold Coast University Hospital and School of Medicine, Griffith University, Southport, Gold Coast, Australia. Dr Liew is affiliated with the School of Information and Communication Technology, Griffith University, Southport, Gold Coast, Australia. Dr Sun is affiliated with Menzies Health Institute Queensland and School of Medicine, Griffith University, Southport, Gold Coast, Australia.

The authors have disclosed no conflicts of interest. ease and its limitations, many patients with COPD experience reduced health-related quality of life (HRQOL). ${ }^{4,5}$ HRQOL involves mental, physical, and social aspects, and is best defined as the individual's perceptions of his or her mental, physical, and social capabilities, and how he or she is impacted by this medical condition over a period of time (Centers for Disease Control and Prevention: HRQOL concepts; https://www.cdc.gov/HRQOL/concept.htm, Accessed May 22, 2018). More specifically, the concept of HRQOL reflects the gap between actual and desired capabilities of

Correspondence: Jing Sun PhD, School of Medicine, Griffith University, Gold Coast campus, Parklands Drive, Southport, QLD, 4222, Gold Coast, Australia. E-mail: j.sun@griffith.edu.au.

DOI: $10.4187 /$ respcare. 05935 
the subject with $\mathrm{COPD}^{6}$ and includes symptomatic experiences and physical and psychosocial functioning. ${ }^{7}$

Research on the characteristics of the HRQOL of subjects with COPD identified various predictors of wellbeing. Blinderman et $\mathrm{al}^{8}$ found that female sex, high symptom distress, greater functional impairments, and poor psychological well-being were associated with having a lower HRQOL. Other studies found social isolation, ${ }^{9}$ other comorbid symptoms, age, marital status, ${ }^{10}$ anxiety, depression, ${ }^{10,11}$ and sex were significant predictors of the HRQOL of the subject with COPD. ${ }^{10,12}$ Although one study recently investigated and found a correlation between the total resilience and quality of life of subjects with COPD, ${ }^{13}$ no studies have yet investigated the HRQOL of subjects with COPD and the individual and contextual resilience factors as a collective concept.

The concept of resilience in the HRQOL of patients with COPD is important because it has been found to influence how subjects manage their health and well-being. ${ }^{14,15}$ Our study conceptualized resilience by using a socio-ecological approach ${ }^{16,17}$ and identified that resilience comprises various individual and contextual factors that facilitate well-being when experiencing stress. ${ }^{18}$ The socio-ecological approach considers the interactions among 3 key areas: that is, individual factors of patients with COPD (eg, self-esteem, self-efficacy, and coping ${ }^{18,19}$ ), their contextual factors (eg, their support systems, including support from family, friends, and their health-care professionals ${ }^{16,18}$ ); and the interactions within their environment, including their relationships, cultural identity, and the material resources available to them. ${ }^{18,19}$ Thus, HRQOL is proposed to be associated with patients' perception of themselves as confident, self-efficiently managing their disease conditions, in addition to their ability to access support and encouragement from professionals and their other relationships.

An individual's resilience has been found to fluctuate over time and can influence decisions to undertake actions that improve their health and well-being. ${ }^{20}$ There is research that identified that subjects with COPD frequently experience low levels of self-esteem, which can impact their motivation and decrease their health and well-being. ${ }^{21}$ Positive use of coping by subjects with COPD has been found to improve HRQOL, ${ }^{22}$ especially when subjects are provided with disease-specific education from health-care professionals. ${ }^{23}$ However, qualitative studies revealed that the benefits of support and relationships for subjects with COPD are unclear, with some studies that support improvements in HRQOL ${ }^{24-26}$ and other studies that report few perceived benefits. ${ }^{25,27}$ Although the value of professional support for improving patients' HRQOL is uncertain, it has been identified that support from family and friends is beneficial. ${ }^{28}$

\section{QUICK LOOK}

\section{Current knowledge}

Declining health-related quality of life (HRQOL) is common in patients with COPD, especially as the disease and its limitations increase. Poor psychological wellbeing is associated with having a lower HRQOL. However, having strong resilience, both individual and contextual has been found to improve an individual's well-being when experiencing stress.

\section{What this paper contributes to our knowledge}

Both individual and contextual resilience factors were important for the HRQOL of the subjects with COPD. Self-esteem, self-efficacy, poor coping, relationships, and belief in professionally supported pulmonary rehabilitation were important for the HRQOL on the breathlessness, symptoms, and cough domains for subjects with COPD. Subjects' recruitment location, marital status, levels of anxiety/depression, and number of comorbidities were also important for HRQOL.

Although some resilience factors have been examined individually for the HRQOL of subjects with COPD, ${ }^{15,23,29-31}$ no studies have comprehensively examined resilience factors at both individual and contextual levels when using a socio-ecological approach. Further, no studies have analyzed the importance of these factors by using a decision tree analysis. The identification of the importance of these resilience factors for each of the HRQOL domains can identify future research directions and potentially inform new practice guidelines for assisting in the treatment and management of the disease. Our study aimed to identify which resilience factors are important in subjects with COPD across a variety of HRQOL domains.

\section{Methods}

\section{Participants}

Data for this study were obtained between September 2015 and November 2016. Based on the Australian prevalence of $8.4 \%$ (https://lungfoundation.com.au/health-pro fessionals/clinical-resources/copd/copd-the-statistics/, Accessed May 22, 2018) and by using the sample size formula devised by Charan and Biswas, ${ }^{32}$ a minimum of 118 participants were required for this research study $(P=8.4 \%$, $\mathrm{d}=0.05$, and $\mathrm{Z}_{1-\alpha / 2}=1.96[P=$ prevalence, $\mathrm{d}=$ absolute error or precision, $Z_{1-\alpha / 2}=$ standard normal variate]). Three hundred and sixty subjects were asked to participate, with 159 completing and returning the questionnaire (44.2\% 
response rate). The participants in this study were $>40 \mathrm{y}$ old, had a physician's diagnosis of COPD conducted via spirometry, were attending COPD education days at Brisbane or Gold Coast Health Community Centres (Helensvale and Robina), or were receiving treatment at various hospital areas, such as the wards or the medical assessment unit at the Gold Coast University and Robina hospitals. Patients were excluded if they had a serious cognitive impairment or any other unstable medical, major psychiatric, and/or neurological conditions. Patients were also excluded if they were unable to speak English or provide informed consent. Ethical approval for this study was gained from both Griffith University Human Research Ethics and the Queensland Health's Gold Coast Hospital and Health Service Human Research Ethics Committee.

\section{Study Measures}

The independent variables included in this study involved a combination of individual and contextual factors that make up resilience. The individual-level factors include self-efficacy, coping, decision-making, defensive coping, and self-esteem, whereas the contextual factors include relationships and professional support. The outcome variable assesses the HRQOL of subjects with COPD by using 5 domains: activity, breathlessness, symptoms, cough, and medication. A comprehensive discussion of the variables in this study is described below (Resilience scale, demographic variables, and HRQOL).

\section{Resilience Scale}

The Connor-Davidson Resilience Scale, ${ }^{7}$ a self-report measure that assesses individual and contextual resilience factors of patients with COPD by using 25 questions. The Connor-Davidson Resilience Scale has an excellent reliability (Cronbach $\alpha=0.89$ ) and good validity. ${ }^{7}$ This questionnaire was used to ask participants to rate their feelings over the last month on a 5-point Likert scale: not at all true, rarely true, sometimes true, often true, and almost always true. Specifically, it queries 4 factors based on factor loadings for the current sample; self-efficacy (can deal with whatever comes), coping (under pressure, I focus and think clearly), decisionmaking (make unpopular or difficult decisions), and relationships (have close and secure relationships). The reliability of the Connor-Davidson Resilience Scale for the current COPD sample was found to be excellent $(\alpha=0.92)$ and content validity for the questions was good, with an accounting of $56.58 \%$ of the variance.

Four items that assessed subjects' use of defensive coping (by denial) and 10 questions that assessed subjects' disease-specific self-esteem were measured by Acceptance of Disease and Impairments Questionnaire. ${ }^{33} \mathrm{~A}$ validation study found the Acceptance of Disease and Impairments
Questionnaire to have good-to-excellent reliability (Cronbach $\alpha$ between 0.80 and 0.83 ) and also proved to be a valid measure. ${ }^{33}$ This questionnaire is used to ask subjects to rate their level of agreement on a 4-point Likert scale: disagree, slightly agree, strongly agree, and entirely agree. It queries patients' level of denial (I try to ignore my impairments) as well as their self-esteem (I feel frustrated with my impairments, I become sad when I experience an impairment, and I can accept my impairments). A reliability of the Acceptance of Disease and Impairments Questionnaire was run with the current sample and was found to have excellent reliability (Cronbach $\alpha=0.865$ ) in addition to having an excellent content validity, with the questions accounting for $78.1 \%$ of the variance.

Subjects' professional support was measured by the Pulmonary Rehabilitation Benefits and Consequences Scale. This scale assesses perceived outcomes of the professionally supported pulmonary rehabilitation programs by patients with COPD. A 7-point Likert scale is used to rate these beliefs: strongly disagree, disagree, somewhat disagree, undecided, somewhat agree, agree, and strongly agree. A factor analysis revealed 3 factors' belief scores: health and well-being (improve a COPD patients' quality of life), reliance on external services (reduce COPD patients' ability to perform basic home duties), and potential risks (increase the risk of injury). The reliability for the Pulmonary Rehabilitation Benefits and Consequences Scale was found to be acceptable $(\alpha=0.72)$, and the content validity was good, with the questions accounting for $59.93 \%$ of the variance.

\section{Demographic Characteristics}

Seven demographic variables were considered in the analysis after a review of the literature. These characteristics included the subjects' marital status, age, sex, smoking status, diagnosis of anxiety or depression, total number of comorbidities, and their recruitment location. The results of the bivariate analysis for the subjects' demographic characteristics are presented in Table 1.

\section{Primary Outcome Measure}

Subjects' HRQOL was the primary outcome measure for this study and was measured by using the St George's Respiratory Questionnaire. ${ }^{34}$ A factor analysis was conducted and factor loadings were calculated for the current sample, with 5 factors being identified. This self-report measures a patient's disease-specific HRQOL (high scores indicate a poor HRQOL and low scores indicate a high HRQOL) by limitations in activity, breathlessness, symptoms, coughing experiences, and medication impacts. The first domain assesses patients' limitations to their daily activities that occur as a result of their breathlessness. The 
RESILIENCE AND HRQOL IN COPD

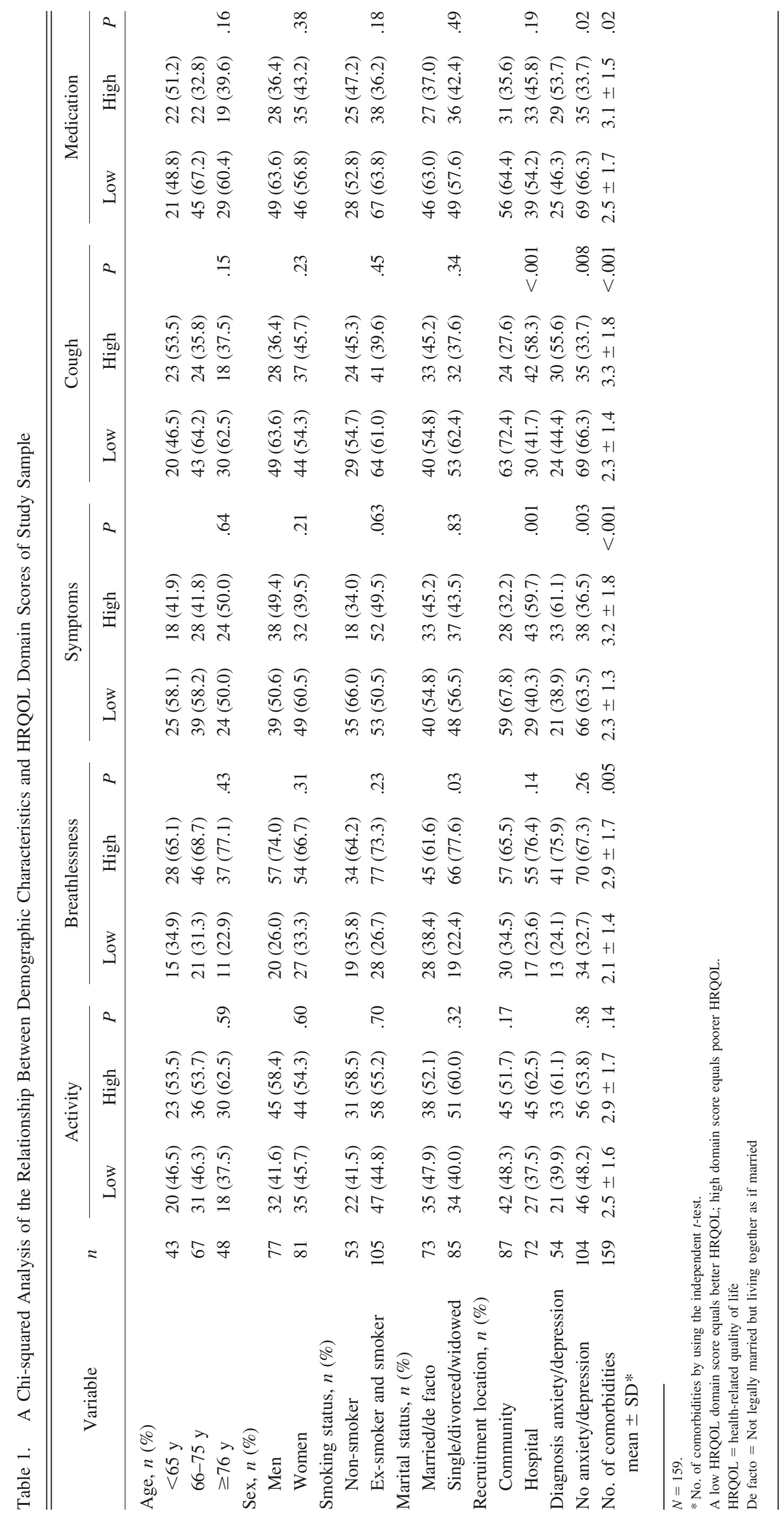


Table 2. Median and Interquartile Range (IQR) for Determining Dichotomisation of Domains

\begin{tabular}{|c|c|c|c|c|c|}
\hline & Activity & Breathlessness & Symptoms & Cough & Medication \\
\hline Median (IQR) & $5(2-7)$ & $9(8-9)$ & $1(0-3)$ & $3(2-4)$ & $0(0-1)$ \\
\hline High HRQOL & $<5$ & $<9$ & $<2$ & $<4$ & $<1$ \\
\hline
\end{tabular}

second factor assesses patients' level of breathlessness currently and in comparison with others. The third factor assesses patients' perception of their symptoms by ratings of their level of breathlessness, cough, and sputum production. The fourth domain examines patients' coughing experiences, and the final factor queries patients' medication experiences. The following factor identification domains were dichotomized into high and low HRQOL owing to the ordinal nature of the data. Cutoff points were determined by analysis of the median (Table 2). The American version of the St George Respiratory Questionnaire was found to have good discriminant and construct validity. The internal reliability $(\alpha>0.70)$ was excellent, and the test-retest correlations were also found to be excellent $\mathrm{r}=0.795$ to $0.900 ;{ }^{35}$ The reliability of the St George Respiratory Questionnaire for the COPD sample obtained for this study was found to be excellent (Cronbach $\alpha=0.89$ ).

\section{Statistical Methods}

Decision tree analysis was conducted to identify the most important resilience factors for HRQOL in subjects with COPD and included possible confounding factors. Decision trees are a useful method for predicting the importance of numerous independent variables and can analyze both continuous (regression tree) and categorical outcome variables classification tree. ${ }^{36}$ In addition to this, the prediction models can be represented by using a decision tree diagram. ${ }^{37}$ The tree analysis is a robust method and can be used with data with missing values, non-homogenous, or not normally distributed. ${ }^{36}$ This tree analysis was conducted with an alpha of 0.05 .

\section{Results}

The study sample consisted of 159 subjects with COPD, of whom, $87(54.7 \%)$ were recruited from the community and 72 were recruited from the Robina and Gold Coast University Hospitals. Of the sample, $51.3 \%$ were women and almost half $(42.4 \%)$ of the participants were ages between 66 and $75 \mathrm{y}$, with a further $30.4 \%$ being $>76 \mathrm{y}$ old. A large proportion of the sample $(n=145$ [91.2\%] $)$ did not smoke, with 92 of the participants $(57.9 \%)$ being ex- smokers; thus only 13 of the recruited participants (8.2\%) continued to smoke. The marital status of the sample was roughly even, with $45.9 \%$ of participants being married or in a de facto relationship. The mean number of comorbid illnesses within the COPD sample was $2.71 \pm$ SD 1.64; one participant reported 8 other comorbidities. Anxiety and depression were common within the sample; with $34.2 \%$ reporting that they had received a diagnosis of anxiety or depression from their physician. Those who had completed a pulmonary rehabilitation program made up $39.1 \%$ of the sample, whereas $26.9 \%$ of the participants had never been referred to a program.

The psychological aspects of the current sample found that most of the participants reached the stage of acceptance (mean 4.02, \pm SD 0.96) within the grief model. However, they still continued to experience strong feelings of anger (resistance mean 3.13, \pm SD 1.05) with regard to their illness and the limitations it placed on their lives. The resilience scores of the sample were strong (Connor-Davidson Resilience Scale total, mean $69.47 \pm$ SD 15.71) with the individual factor scores being relatively consistent across each of the factor loadings. Also, the HRQOL of the current COPD sample revealed moderate-to-low HRQOL across 3 domains and for their overall well-being (activity mean 4.25, \pm SD 2.47; breathlessness mean 8.12, \pm SD 1.84; cough mean 3.10, \pm SD 1.98; and total mean 56.25 , \pm SD 17.16), yet high HRQOL for the remaining 2 domains were symptoms mean 1.89, \pm SD 2.04, and for medication were mean $0.74, \pm$ SD 1.42 .

Bivariate analyses (Table 1) found numerous potential confounding relationships with the HRQOL domains (marital status, recruitment location, diagnosis of anxiety or depression) and total number of comorbidities. These potential confounds were thus included in decision tree analysis. The results of the decision tree identified the factors that are of importance for the HRQOL of subjects with COPD. On the HRQOL breathlessness domain, 7 resilience factors and 2 confounding factors were found to be important for the levels of breathlessness of the subjects with COPD (Fig. 1). The most important factor on the breathlessness domain was subjects' marital status. On the first branch of the decision tree, the subjects with COPD who were married or de facto (Not legally married but living together as if married) and used defensive coping strategies, had high self-esteem, believed that supported pulmonary rehabilitation would improve their health and well-being, and had one or no other comorbidities were found to have high HRQOL. High HRQOL was also found for single, divorced, or widowed subjects with COPD who had high levels of coping, good decision making abilities, high self-efficacy, and $<2$ comorbidities. The overall prediction accuracy for the HRQOL symptoms decision tree analysis was reasonable, at $62.26 \%$ (Table 3). 


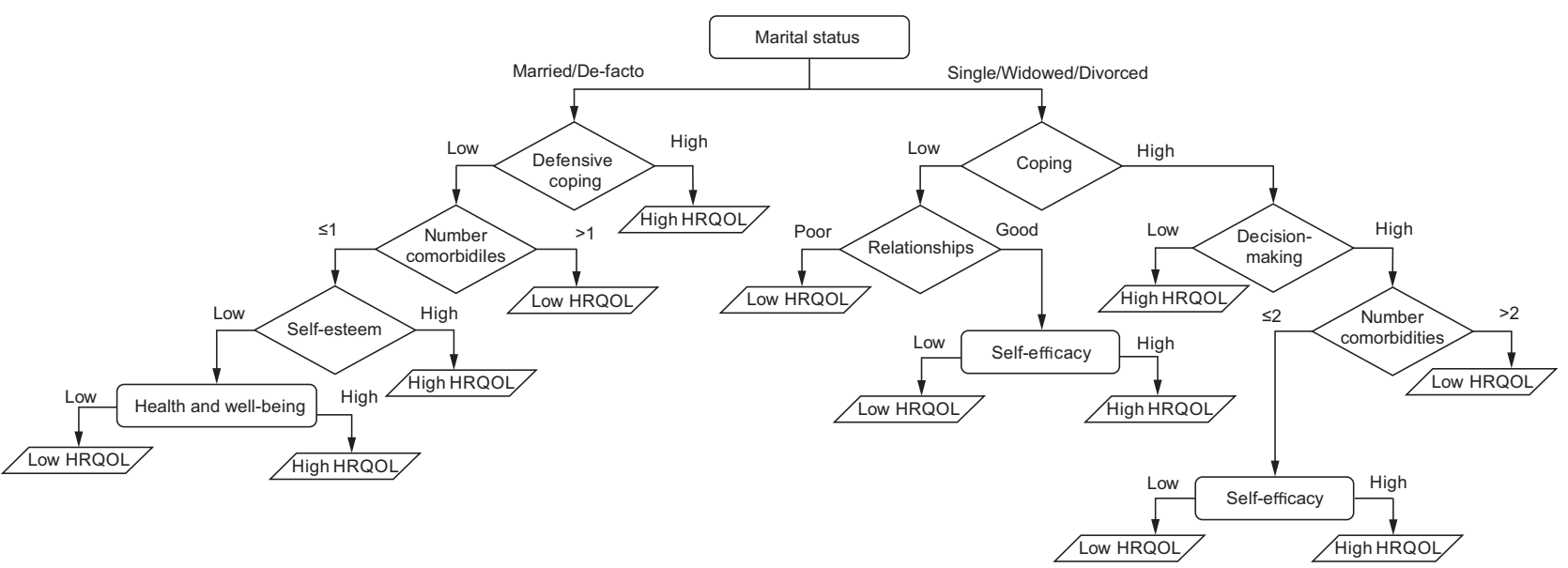

Fig. 1. Decision tree diagram that indicates resilience pathways important to the health-related quality of life (HRQOL) of the subjects with COPD on the breathlessness domain. Subject HRQOL breathlessness scores are presented as either high or low for each outcome for the corresponding resilience or confounding factor. Resilience factor scores are presented as to whether subjects with COPD scored high or low on that factor.

Table 3. Decision Tree Analysis Accuracy by Class for the Breathlessness, Symptoms, and Cough Domains

\begin{tabular}{lccc}
\hline \hline \multicolumn{1}{c}{ Class } & True Positive Rate & Precision & ROC Area \\
\hline Breathlessness & & & \\
High HRQOL & 0.30 & 0.34 & 0.51 \\
Low HRQOL & 0.76 & 0.72 & 0.51 \\
$\quad$ Total & 0.62 & 0.61 & 0.51 \\
Symptoms & & & \\
$\quad$ High HRQOL & 0.59 & 0.59 & 0.53 \\
Low HRQOL & 0.49 & 0.49 & 0.53 \\
$\quad$ Total & 0.55 & 0.55 & 0.53 \\
Cough & & & \\
$\quad$ High HRQOL & 0.82 & 0.66 & 0.59 \\
Low HRQOL & 0.39 & 0.61 & 0.59 \\
Total & 0.64 & 0.63 & 0.59 \\
& & & \\
ROC $=$ receiver operating characteristic & & \\
HRQOL = health-related quality of life & & \\
\hline
\end{tabular}

The decision tree results for the HRQOL symptoms domain found that 7 resilience factors and 2 confounding factors were important in the perception of their symptoms in subjects with COPD (Fig. 2). The most important factor on the symptoms domain was the level of self-efficacy of the subjects with COPD; poor self-efficacy directly determined poor HRQOL. However, on the symptoms domain, high HRQOL was found in the subjects with a high level of self-efficacy and high self-esteem when the subjects had no anxiety and depression, and were recruited from the community. When the subjects had low self-efficacy and low coping but good relationships and high beliefs that supported pulmonary rehabilitation would improve their health and well-being, they also were found to have a high HRQOL on the symptoms domain. Hospitalized subjects with high self-efficacy, good decision making abilities, high levels of coping even though they were diagnosed with anxiety and depression also displayed high HRQOL. The overall prediction accuracy for the HRQOL symptoms decision tree analysis was found to be reasonable, at $54.72 \%$.

The third HRQOL domain to have important factors was the cough domain (Fig. 3). The decision tree analysis found that 3 resilience factors and 2 confounding factors were important for the coughing experiences of the subjects with COPD. The most important factor was the recruitment location of the subject, with the subjects recruited from the community having a better HRQOL. Subjects with COPD who were recruited from the hospital had a better HRQOL if they did not have anxiety or depression, and had high beliefs in supported pulmonary rehabilitation improving their well-being. In addition to this, the subjects with COPD with good coping abilities who used defensive coping were also able to maintain a good HRQOL. This was also true for subjects who had a diagnosis of anxiety or depression yet had good coping skills. The HRQOL symptoms decision tree analysis overall prediction accuracy was reasonable, at $64.15 \%$. The outcome variables activity and medication did not find any resilience factors or confounds to be important in the HRQOL of subjects with COPD.

\section{Discussion}

The decision tree analysis identified associations with both individual- and contextual-level resilience factors and HRQOL in the subjects with COPD. With the exception of the professional support subvariable reliance on external services, all individual and contextual resil- 


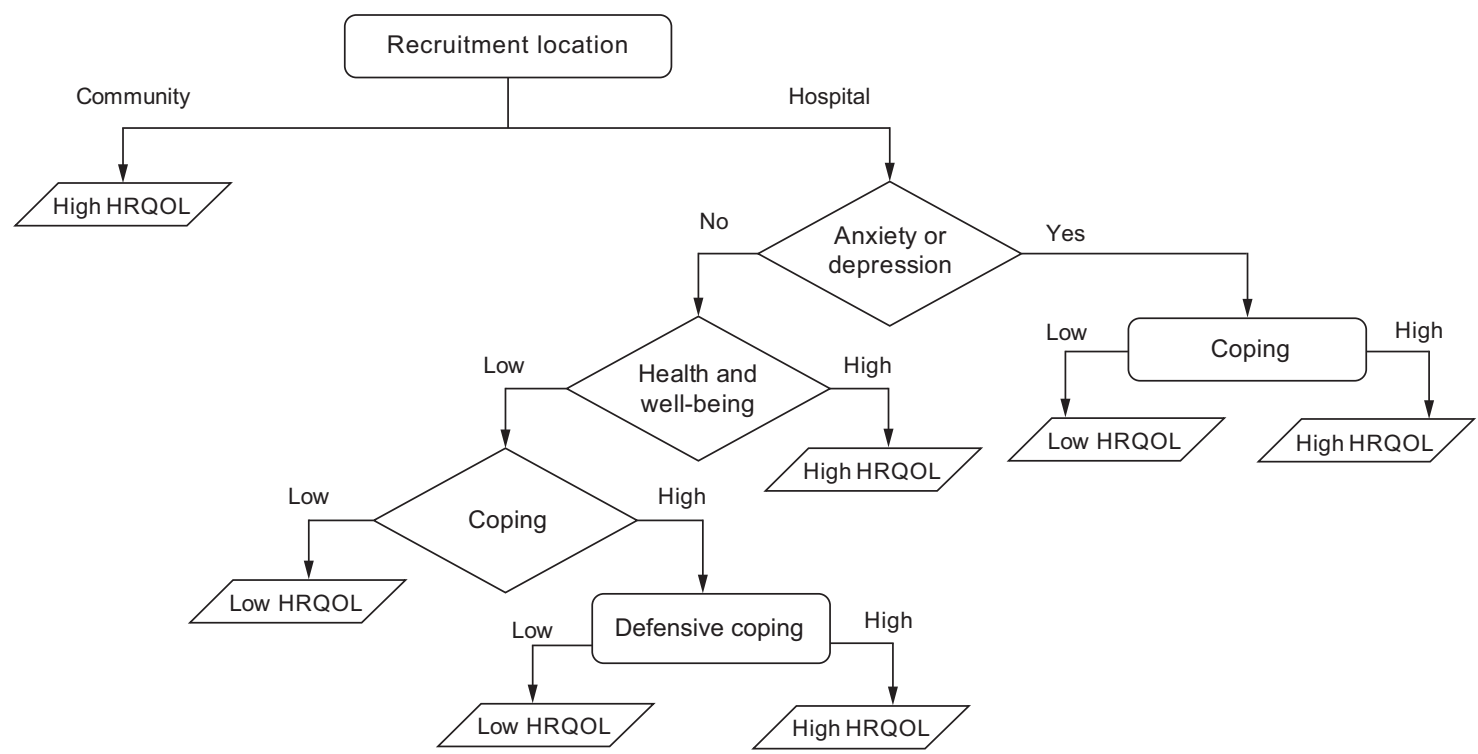

Fig. 2. Decision tree diagram that indicates resilience pathways important to health-related quality of life (HRQOL) of the subjects with COPD on the symptoms domain. The HRQOL symptoms scores of the subjects with COPD are presented as either high or low for each outcome for the corresponding resilience or confounding factor. Resilience factor scores are presented as whether the subjects with COPD scored high or low on that factor.

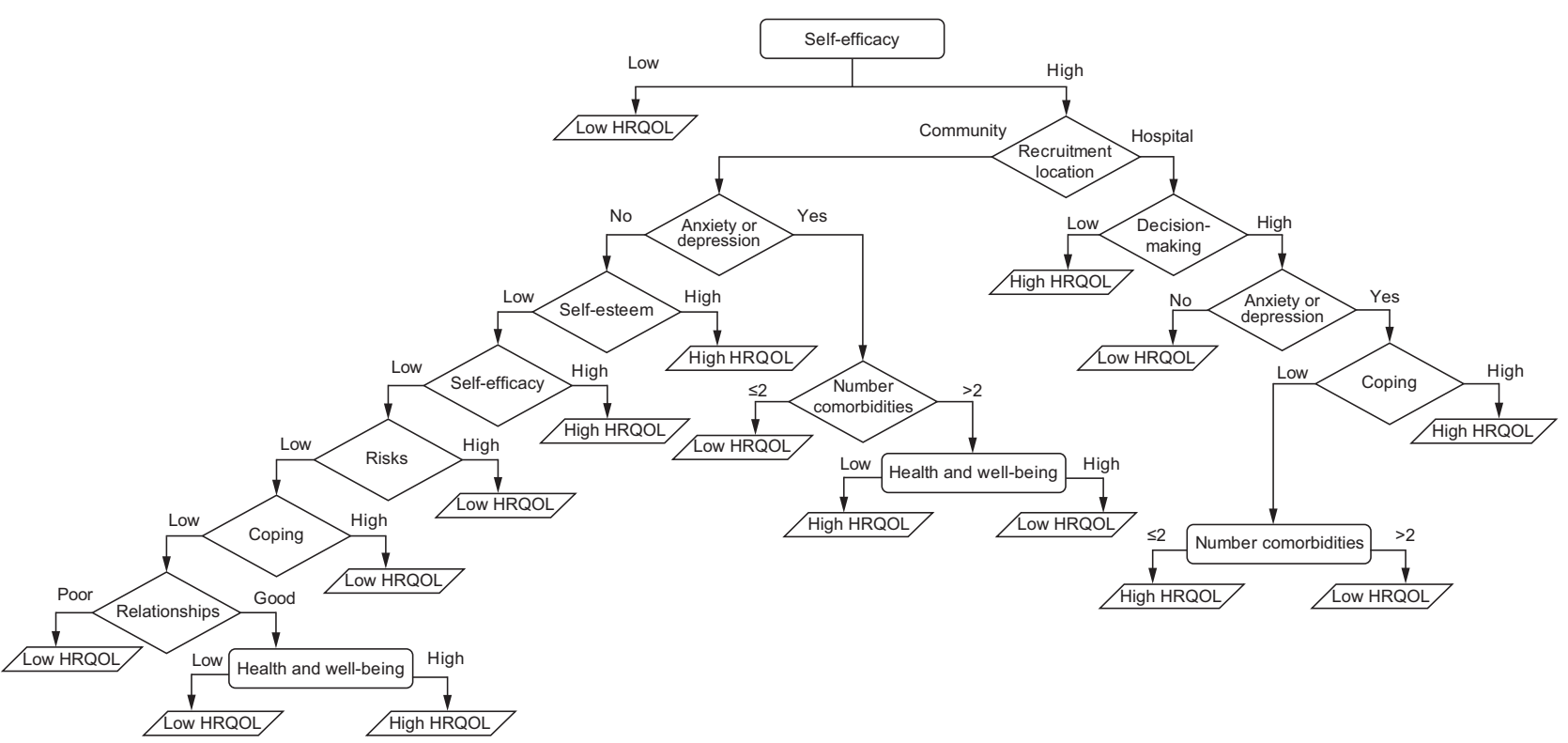

Fig. 3. Decision tree diagram that indicates resilience pathways important to the health-related quality of life (HRQOL) on the cough domain of the subjects with COPD. The HRQOL cough scores of subjects with COPD are presented as either high or low for each outcome for the corresponding resilience or confounding factor. Resilience factor scores are presented as to whether the subjects with COPD scored high or low on that factor.

ience factors were found to be associated with the HRQOL of subjects with COPD throughout the breathlessness, symptoms, and/or cough domains. When considering the confounding factors, the subjects' recruitment location, number of comorbidities, marital status, and diagnosis of anxiety or depression were found to be important with some of the HRQOL domains.

\section{HRQOL-Related Breathlessness}

The current breathlessness experiences of subjects with COPD were found to be associated with their levels of resilience when marital status and comorbidities were controlled for in the model. Similar results were found in other studies, in which subjects with COPD who were educated 
in breathing techniques were found to become empowered, which increased their self-efficacy and left them feeling better equipped to cope, including in making decisions regarding potential episodes of dyspnea. ${ }^{38,39}$ Interventions that provided breathing techniques were found to increase the beliefs of subjects with COPD that professionally supported programs were able to improve their health and well-being. ${ }^{39}$ These programs further promoted the idea that patients would require assistance from family and friends with some tasks, especially when their dyspnea was high, ${ }^{38}$ a finding that is consistent with our current results that good relationships are important for HRQOL (breathlessness and symptoms).

The association between the HRQOL of patients with COPD and their use of defensive coping has been little studied in COPD, yet has had inconsistent results in other HRQOL chronic illness research. These studies found that the use of defensive coping was significantly related to poorer HRQOL ${ }^{40}$ and significantly higher rates of emergency department visits over a period of 12 months. ${ }^{41}$ In contrast to this, another study found that defensive coping was associated with marginally better physical HRQOL. ${ }^{42}$ Thus, defensive coping may act as a buffer against the harsh reality of the illness and its consequences, and provide short-term relief from the distress caused by having a chronic illness, for example, COPD. ${ }^{42}$

\section{HRQOL-Related Symptoms}

The subjects' perception of their symptomatic experiences was found to have various complex associations with both resilience and confounding factors. Although many of these associations have not yet been directly studied, these findings were consistent with similar previous chronic illness research. ${ }^{29,43}$ The subjects who had higher baseline self-efficacy were found to exhibit significant improvements in HRQOL after a pulmonary rehabilitation program..$^{29}$ Other research found that subjects who had been admitted to the hospital with multiple exacerbations had a worse perception of their symptoms than subjects who had exacerbations for which they were not hospitalized. ${ }^{43}$

The subsequent associations with subjects' symptomatic HRQOL found in this study have been similarly identified in other research. First, high self-esteem was found to be associated with subjects who experienced fewer symptoms, for example, sputum production. ${ }^{44}$ Second, high selfesteem in subjects with COPD has also being found to be associated with better relationships and coping abilities; aspects that are associated with fewer COPD symptoms. ${ }^{15}$ Positive professional support, in which patients believe that the support will improve their health and well-being is recognized as increasing adherence to programs that improve subjects' HRQOL. ${ }^{45}$ However, if patients believed that the program would worsen their symptoms, they refused to participate in these programs, which reduced the probability of improving their symptom management and HRQOL. ${ }^{46}$ Also, the number of comorbidities that subjects had was found to influence the HRQOL symptoms of subjects with COPD, in which subjects with fewer comorbidities were found to have a better HRQOL. ${ }^{6}$

Unexpected findings included that hospitalized subjects with COPD and poor decision making had high HRQOL and subjects without anxiety or depression ranked with poor HRQOL. When considering subjects with poor decision making, it was expected that poor choices would lead to poorer HRQOL. However, there is research that indicates that seemingly poor decisions could actually provide the patient with some relief; for instance, being less adherent with medications to avoid adverse effects. ${ }^{47}$ The finding that subjects with COPD without depression had a low symptomatic HRQOL could be explained by research, which found that the subjects admitted to the hospital tend to experience higher rates of depressed feelings during their admission, exacerbating the perception of their symptoms until these feelings dissipate, usually 12 weeks after discharge. ${ }^{48}$

\section{HRQOL-Related Cough}

The finding that the recruitment location of subjects with COPD was associated with their coughing experiences was consistent with previous research. ${ }^{15,44,49}$ First, high levels of coughing and sputum production are associated with the exacerbations that frequently lead to hospitalizations. ${ }^{49}$ In addition to this, the primary cause of exacerbations and acute coughing experiences usually results from a bacterial or viral respiratory infection, ${ }^{50}$ which are disturbances that are strongly associated with hospitalizations, poor well-being, and increased mortality rates. ${ }^{51}$

The second association, that anxiety and depression are important in subjects' HRQOL cough, has been established in previous studies in which depression or anxiety was found to be significantly related to low HRQOL in subjects with COPD. ${ }^{52-54}$ It is also recognized that anxiety is the factor that is most strongly related to patients' breathlessness. One study found that a severe bout of coughing can create dyspnea, which increases subjects' anxiety, ${ }^{53}$ and anxiety can also cause breathlessness, ${ }^{54}$ which can promote the action of coughing to gain better lung clearance. ${ }^{55}$ It was also found that subjects with COPD who believe that professional support will improve their health and well-being had a higher HRQOL on the cough domain. This finding is supported by research that investigated subjects with COPD after pulmonary rehabilitation interventions; ${ }^{45}$ these patients were better able 
to cope with their disease by managing their breathlessness and coughing experiences following their training, providing them with more independence and a better HRQOL. ${ }^{45}$

Resilience factors for the HRQOL domains of medication and activity were found to be unimportant in this COPD sample. The influence of resilience on medication-based HRQOL is under-researched in patients with COPD, with the closest research to consider the relationships of resilience factors and medication adherence being in other chronic illnesses. One paper identified that self-efficacy and support could be factors that influence medication adherence in patients with chronic illness. ${ }^{56}$ Another paper, which investigated adherence in a psychiatric population, found that, when medication interfered with resilience and resilience supporting factors, the subjects' adherence decreased to preserve their current state. ${ }^{57}$

When considering the insignificant association between HRQOL activity and individual and contextual resilience factors of subjects with COPD, one similar publication found that the quality of life on physical health of subjects with COPD was correlated with their overall resilience. ${ }^{13}$ However, the physical health score used in this study contained a diverse array of items that were not directly related to our activity score, including pain, fatigue, and sleep. Because our paper opposes these past findings, more research on the importance of resilience factors for the HRQOL of patients with COPD is required to clarify these outcomes.

\section{Implications}

The results of this study demonstrated that individual and contextual resilience factors of the subjects with COPD were associated with their HRQOL. It was also found that various demographic factors influenced the results. Subjects' belief in their own self-esteem and self-efficacy have been recognized as important factors in coping with the adverse experiences and lifestyle changes that are required after the diagnosis of a chronic illness. ${ }^{5,58}$ Individuals who have low levels of self-esteem and self-efficacy tend to have more negative appraisals of their situation and, as a result, are less adept at managing stressful situations..21 Aspects that have been identified as impairing the selfesteem and self-efficacy of patients with COPD is their ability to control their situation, having feelings of guilt over causing their illness, and their need for consistent complex medication regimens. ${ }^{59}$ In addition to this, it has also been identified that subjects' with a mental illness experience continual declines in self-esteem after onset and diagnosis of their illness. ${ }^{60}$ This finding is important for patients with COPD because anxiety and depression are common comorbidities in COPD and can thus lead to further deterioration of subjects' self-esteem, self-efficacy, and COPD symptoms. ${ }^{5}$

To improve the HRQOL of patients with COPD, the patients need to be able to hold positive views of their own self-worth and have competence for managing their disease. The patients' feelings regarding their illness need to be shifted from a perspective of culpability, shame, and guilt, to providing feelings of empowerment and empathetical support, both from professionals and their external relationships. Patients frequently feel disempowered and unworthy of care after hospitalization, ${ }^{27}$ and, because subjects recruited from the hospital who had poor coping abilities and other comorbidities, primarily anxiety or depression, were found to experience a poorer HRQOL in this study, it is important to consider both the medical and psychological aspects of the patient at discharge.

Promoting self-esteem, self-efficacy, and coping can be achieved through the identification of patients' health goals and encouraging the use of positive health behaviors to achieve these goals. ${ }^{61}$ There is research that found that the approval of these positive health behaviors from a health-care provider promotes stronger and more enduring changes in the subjects' self-esteem (Vonk et al 2004 as cited in Vonk et al ${ }^{62}$ ). In a COPD context, the enhancement of patients' resilience would be best achieved through the identification and encouragement of positive health behaviors, such as exercise, medication adherence, and early exacerbation identification. Identification of these behaviors can be achieved during consults with medical professionals (eg, general practitioners or specialists) or during hospital discharge by a respiratory nurse, with referral to intervention programs being made to further these skills if necessary.

\section{Limitations and Recommendations}

Although this study highlighted some positive findings, it had a number of limitations that needed to be considered when interpreting the results. First, this study relied on data that were obtained by using a self-reported questionnaire, which is a subjective measure and is based on the individual's perspective. As a result, the answers provided in the questionnaire may not objectively reflect the real experiences or severity of subjects who are undergoing extreme difficulties. In addition to this, all the participants in this study were recruited from 2 regions (Brisbane and Gold Coast) in South East Queensland. In recruiting participants in this way, it may not be possible to generalize the results to other areas of Australia and other international cultural locations. A third limitation involved the recruitment of community participants; that is, that all the community participants were recruited from COPD education seminars. Recruiting participants from such loca- 
tions indirectly excluded non-hospitalized participants who were not actively undertaking education. Participants not undertaking education could be in denial about their illness or may not be motivated enough to undertake such courses, an aspect that could impact the outcome of the results. Also, because the study was cross-sectional, it only allowed for the interpretation of relationships among the variables and did not provide the opportunity to assess any causal conclusions. Thus, future replications should look at obtaining data from large randomized controlled trials to examine the cause and effect relationship between resilience and HRQOL, in addition to being expanded to other areas to contain a more representative sample to assist in generalizability of the results across the COPD population.

\section{Conclusions}

Individual and contextual resilience and confounding factors in subjects with COPD were found to be important in their HRQOL. Specifically, a high HRQOL was found in subjects who had high levels of self-esteem, self-efficacy, good coping, relationships, and positive views of supported pulmonary rehabilitation programs. Recruitment location, marital status, levels of anxiety/depression, and number of comorbidities of subjects with COPD were also found to be associated with the HRQOL, and these factors were controlled for in the models. These findings indicated that resilience is important in subjects with COPD who had other comorbidities, especially after being admitted to the hospital. Thus, patients with COPD require identification, encouragement, and approval of their current disease management abilities from a health practitioner, especially at the time of hospital discharge to improve their resilience and, ultimately, HRQOL. Furthermore, new treatment recommendations and referrals to intervention programs need to be conveyed in a way that aligns with the individual's goals and beliefs to maintain the patient's resilience.

\section{ACKNOWLEDGMENTS}

The authors thank Dr Siddharth Sharma and Kate Harland at the Gold Coast University Hospital for their contributions, and to Rose Costa and the community respiratory service multidisciplinary team at Robina and Helensvale for providing support and access for the data collection of this project. A special thank you to Garry Power and Sen Wang for their assistance with refining the statistical analysis.

\section{REFERENCES}

1. GOLD. Global strategy for the diagnosis, management and prevention of COPD. Global Initiative for Chronic Obstructive Lung Disease (GOLD); 2016 Available from: https://goldcopd.org.
2. Abramson M, Crockett AJ, Dabscheck E, Frith PA, George J, Glasgow N, et al. The COPD-X Plan: Australian and New Zealand Guidelines for the management of Chronic Obstructive Pulmonary Disease V2.43, Sept 2015. 2015; v2.43 Available from http:// www.copdx.org.au/the-copd-x-plan-pdf.

3. Uchmanowicz I, Jankowska-Polanska B, Chabowski M, Uchmanowicz B, Fal AM. The influence of frailty syndrome on acceptance of illness in elderly patients with chronic obstructive pulmonary disease. Int J Chron Obstruct Pulmon Dis 2016;11:2401-2407.

4. Cully JA, Graham DP, Stanley MA, Ferguson CJ, Sharafkhaneh A, Souchek J, Kunik ME. Quality of life in patients with chronic obstructive pulmonary disease and comorbid anxiety or depression. Psychosomatics 2006;47(4):312-319.

5. Uchmanowicz I, Jankowska-Polanska B, Motowidlo U, Uchmanowicz B, Chabowski M. Assessment of illness acceptance by patients with COPD and the prevalence of depression and anxiety in COPD. Int J Chron Obstruct Pulmon Dis 2016;11:963-970.

6. Almagro P, Castro A. Helping COPD patients change health behavior in order to improve their quality of life. Int $\mathrm{J}$ Chron Obstruct Pulmon Dis 2013;8:335-345.

7. Connor KM, Davidson JR. Development of a new resilience scale: the Connor-Davidson Resilience Scale (CD-RISC). Depress Anxiety 2003;18(2):76-82.

8. Blinderman CD, Homel P, Billings JA, Tennstedt S, Portenoy RK. Symptom distress and quality of life in patients with advanced chronic obstructive pulmonary disease. J Pain Symptom Manage 2009;38(1): 115-123.

9. Gardiner C, Gott M, Payne S, Small N, Barnes S, Halpin D, et al. Exploring the care needs of patients with advanced COPD: an overview of the literature. Respir Med 2010;104(2):159-165.

10. Mewes R, Rief W, Kenn K, Ried J, Stenzel N. Psychological predictors for health-related quality of life and disability in persons with chronic obstructive pulmonary disease (COPD). Psychol Health 2016; 31(4):470-486.

11. Keil DC, Vaske I, Kenn K, Rief W, Stenzel NM. With the strength to carry on. Chron Respir Dis 2017;14(1):11-21.

12. Raherison C, Tillie-Leblond I, Prudhomme A, Taillé C, Biron E, Nocent-Ejnaini C, et al. Clinical characteristics and quality of life in women with COPD: An observational study. BMC Womens Health 2014;14(1):31.

13. Sanyal N, Fernandes T, Fathi M, Khatoon S. Illness perception, medication adherence, resilience and quality of life among COPD and arthritis patients. Indian J Health Wellbeing 2017;8(1):18.

14. Hesselink AE, Penninx BW, Schlösser MA, Wijnhoven HA, van der Windt DA, Kriegsman DM, van Eijk JT. The role of coping resources and coping style in quality of life of patients with asthma or COPD. Qual Life Res 2004;13(2):509-518.

15. Juth V, Smyth JM, Santuzzi AM. How do you feel? Self-esteem predicts affect, stress, social interaction, and symptom severity during daily life in patients with chronic illness. J Health Psychol 2008; 13(7):884-894.

16. Bronfenbrenner U. Ecological models of human development. In International Encyclopedia of Education 1994 (Vol. 3, 2nd ed.). Oxford: Elsevier.

17. Sun J, Stewart D. Development of population-based resilience measures in the primary school setting. Health Educ 2007;107(6):575599.

18. Ungar M, Ghazinour M, Richter J. Annual research review: What is resilience within the social ecology of human development? J Child Psychol Psychiatry 2013;54(4):348-366.

19. Wang P, Liu DZ, Zhao X. The social ecology of resilience: A comparison of Chinese and Western researches. Procedia Soc Behav Sci 2014;116:3259-3265. 


\section{RESILIENCE AND HRQOL IN COPD}

20. Bourbeau J, Nault D, Dang-Tan T. Self-management and behaviour modification in COPD. Patient Educ Couns 2004;52(3):271-277.

21. Reich JW, Zautra AJ, Hall JS. Handbook of adult resilience. London: Guilford Press; 2010.

22. Andenaes R, Kalfoss MH, Wahl AK. Coping and psychological distress in hospitalized patients with chronic obstructive pulmonary disease. Heart Lung 2006;35(1):46-57.

23. Cannon D, Buys N, Sriram KB, Sharma S, Morris N, Sun J. The effects of chronic obstructive pulmonary disease self-management interventions on improvement of quality of life in COPD patients: A meta-analysis. Respir Med 2016;121:81-90.

24. Harris D, Hayter M, Allender S. Improving the uptake of pulmonary rehabilitation in patients with COPD: qualitative study of experiences and attitudes. Br J Gen Pract 2008;58(555):703-710.

25. Bulley C, Donaghy M, Howden S, Salisbury L, Whiteford S, Mackay E. A prospective qualitative exploration of views about attending pulmonary rehabilitation. Physiother Res Int 2009;14(3): 181-192.

26. Haave E, Hyland M. Different short-term and longitudinal results on perceived health status for asthma and COPD patients after pulmonary rehabilitation. Patients living alone have the largest improvements in perceived quality of life. Chron Respir Dis 2008; $5(2): 69-73$.

27. Harrison SL, Robertson N, Apps L, C. Steiner M, Morgan MD, Singh SJ. "We are not worthy"- understanding why patients decline pulmonary rehabilitation following an acute exacerbation of COPD. Disabil Rehabil 2015;37(9):750-756.

28. Greeff AP, Holtzkamp J. The prevalence of resilience in migrant families. Family Community Health 2007;30(3):189-200.

29. Bentsen SB, Wentzel-Larsen T, Henriksen AH, Rokne B, Wahl AK. Self-efficacy as a predictor of improvement in health status and overall quality of life in pulmonary rehabilitation-an exploratory study. Patient Educ Couns 2010;81(1):5-13.

30. Ezeamama AE, Elkins J, Simpson C, Smith SL, Allegra JC, Miles TP. Indicators of resilience and healthcare outcomes: findings from the 2010 health and retirement survey. Qual Life Res 2016;25(4): 1007-1015.

31. Adegbola M. Spirituality, self-efficacy, and quality of life among adults with sickle cell disease. South Online J Nurs Res 2011;11(1): pii: 5 .

32. Charan J, Biswas T. How to calculate sample size for different study designs in medical research? Indian J Psychol Med 2013;35(2):121126.

33. Boer LM, Daudey L, Peters JB, Molema J, Prins JB, Vercoulen JH. Assessing the stages of the grieving process in chronic obstructive pulmonary disease (COPD): validation of the Acceptance of Disease and Impairments Questionnaire (ADIQ). Int J Behav Med 2014; 21(3):561-570.

34. Jones PW, Quirk FH, Baveystock CM. The St George's Respiratory Questionnaire. Respir Med 1991;85:25-31; discussion 33-37.

35. Barr JT, Schumacher GE, Freeman S, LeMoine M, Bakst AW, Jones PW. American translation, modification, and validation of the St. George's Respiratory Questionnaire. Clin Ther 2000;22(9):11211145 .

36. Ming ZY. Classification and regression trees (a statistical method suitable for clinical researches). J Beijing Med Univ 2001;6:22.

37. Loh WY. Classification and regression trees. Wiley Interdiscip Rev Data Min Knowl Discov 2011;1(1):14-23.

38. Milne L, Moyle W, Cooke M. Hope: a construct central to living with chronic obstructive pulmonary disease. Int J Older People Nurs 2009;4(4):299-306

39. Vercoulen JH. A simple method to enable patient-tailored treatment and to motivate the patient to change behaviour. Chron Respir Dis 2012;9(4):259-268
40. Carvalho AF, Ramírez SP, Macêdo DS, Sales PM, Rebouças JC, Daher EF, Hyphantis TN. The psychological defensive profile of hemodialysis patients and its relationship to HRQOL. J Nerv Ment Dis 2013;201(7):621-628.

41. González-Freire B, Vázquez-Rodríguez I, Marcos-Velázquez P, de la Cuesta CG. Repression and coping styles in asthmatic patients. J Clin Psychol Med Settings 2010;17(3):220-229.

42. Kaltsouda A, Skapinakis P, Damigos D, Ikonomou M, Kalaitzidis R, Mavreas V, Siamopoulos KC. Defensive coping and HRQOL in chronic kidney disease: a cross-sectional study. BMC Nephrol 2011; 12(1):28.

43. Esteban C, Quintana JM, Moraza J, Aburto M, Egurrola M, España PP, et al. Impact of hospitalisations for exacerbations of COPD on health-related quality of life. Respir Med 2009;103(8): 1201-1208.

44. Nicolson P, Anderson P. Quality of life, distress and self-esteem: a focus group study of people with chronic bronchitis. Br J Health Psychol 2003;8(Pt 3):251-270.

45. Thorpe O, Johnston K, Kumar S. Barriers and enablers to physical activity participation in patients with COPD: a systematic review. J Cardiopulm Rehabil Prev 2012;32(6):359-369.

46. Guo SE, Bruce A. Improving understanding of and adherence to pulmonary rehabilitation in patients with COPD: a qualitative inquiry of patient and health professional perspectives. PLoS One 2014;9(10):e110835.

47. Ágh T, Dömötör P, Bártfai Z, Inotai A, Fujsz E, Mészáros Á. Relationship between medication adherence and health-related quality of life in subjects with COPD: a systematic review. Respir Care 2015;60(2):297-303.

48. Hill K, Geist R, Goldstein RS, Lacasse Y. Anxiety and depression in end-stage COPD. Eur Respir J 2008;31(3):667-677.

49. Burgel PR, Nesme-Meyer P, Chanez P, Caillaud D, Carré P, Perez T, Roche N; Initiatives Bronchopneumopathie Chronique Obstructive (BPCO) Scientific Committee. Cough and sputum production are associated with frequent exacerbations and hospitalizations in COPD subjects. Chest 2009;135(4):975-982

50. Boixeda R, Rabella N, Sauca G, Delgado M, Martínez-Costa X, Mauri M, et al. Microbiological study of patients hospitalized for acute exacerbation of chronic obstructive pulmonary disease (AECOPD) and the usefulness of analytical and clinical parameters in its identification (VIRAE study). Int J Chron Obstruct Pulmon Dis 2012; 7:327-325.

51. Burgel PR. Chronic cough and sputum production: a clinical COPD phenotype? Eur Respir J 40(1):4-6, 2012.

52. Burgel PR, Escamilla R, Perez T, Carré P, Caillaud D, Chanez P, et al; Initiatives BPCO Scientific Committee. Impact of comorbidities on COPD-specific health-related quality of life. Respir Med 2013; 107(2):233-241.

53. Willgoss TG, Yohannes AM, Goldbart J, Fatoye F. "Everything was spiraling out of control": experiences of anxiety in people with chronic obstructive pulmonary disease. Heart Lung 2012; 41(6):562-571.

54. Bailey PH. The dyspnea-anxiety-dyspnea cycle-COPD patients' stories of breathlessness: "It's scary/when you can't breathe". Qual Health Res 2004;14(6):760-778.

55. Yang IA, Dabscheck E, George J, Jenkins S, McDonald CF, McDonald V, et al. The COPD-X Plan: Australian and New Zealand Guidelines for the management of Chronic Obstructive Pulmonary Disease Version 2.50, Jun 2017. 2017; Version 2.50 Available from http://copdx.org.au/copd-x-plan/.

56. Lareau SC, Yawn BP. Improving adherence with inhaler therapy in COPD. Int J Chron Obstruct Pulmon Dis 2010;5:401-406. 


\section{RESILIENCE AND HRQOL IN COPD}

57. Deegan PE. The importance of personal medicine: A qualitative study of resilience in people with psychiatric disabilities. Scand J Public Health Suppl 2005;66:29-35.

58. Kralik D, Van Loon A, Visentin K. Resilience in the chronic illness experience. Educational Action Res 2006;14(2):187-201.

59. Lindqvist G, Hallberg LR. 'Feelings of guilt due to Self-inflicted Disease' a grounded theory of suffering from Chronic Obstructive Pulmonary Disease (COPD). J Health Psychol 2010;15(3):456466.
60. Wittmann D, Smith P, Rajarethinam R, Foley S, VanBrussel A, Phillip $\mathrm{B}$, et al. Do patients with psychosis experience loss and grief as a result of their illness? Psychoanalytic Soc Work 2010;17(1):30-39.

61. Vonk R, Smit H. Optimal self-esteem is contingent: Intrinsic versus extrinsic and upward versus downward contingencies. Eur J Pers 2012;26(3):182-193.

62. Vonk R. Improving self-esteem. Self-esteem issues and answers: A sourcebook of current perspectives: New York, Psychology Press, 2006; 178-187. 